\title{
MAPPINGS OF FINITE DISTORTION: FORMATION OF CUSPS
}

\author{
Pekka Koskela and Juhani Takkinen
}

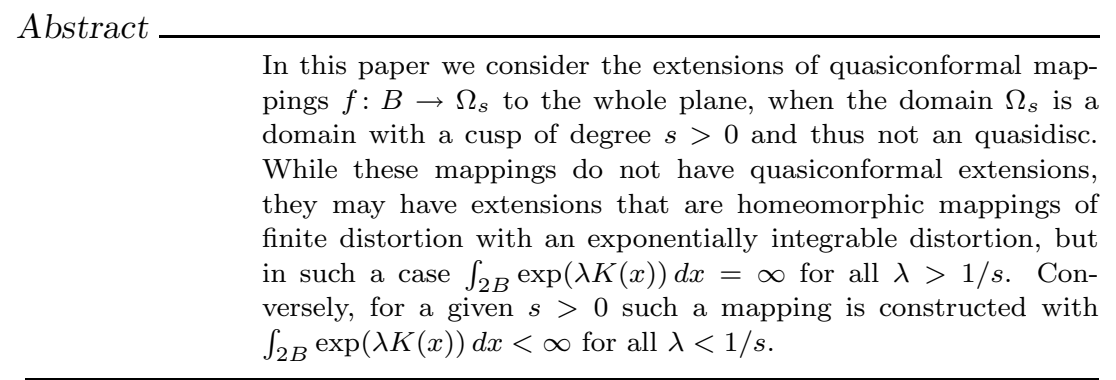

\section{Introduction}

Suppose that $f$ is a quasiconformal mapping of the unit disc $B$ into $\mathbb{R}^{2}$. It is well-known that $f$ has an extension to a quasiconformal homeomorphism of the entire plane if and only if the boundary of $f(B)$ is a quasicircle. Since quasicircles are exactly the Jordan curves which satisfy the so-called three point condition, the extendability of $f$ to a quasiconformal mapping of the entire plane is determined by the geometric properties of the boundary of $f(B)$. For this see $[\mathbf{2}]$. On the other hand, a quasiconformal mapping $f$ of the unit disc into $\mathbb{R}^{2}$ has an extension to a homeomorphism of the entire plane if and only if the boundary of $f(B)$ is a Jordan curve. One is then motivated to ask for a class of homeomorphisms larger than the class of quasiconformal mappings for which the extension property would still be determined by a geometric property. A natural candidate is the class of homeomorphisms of (locally) exponentially integrable distortion introduced by David [1] .

2000 Mathematics Subject Classification. 30C60, 30C62.

Key words. Cusp, mappings of finite distortion, quasiconformal, quasidisc.

Juhani Takkinen was partially supported by the foundation Vilho, Yrjö ja Kalle Väisälän rahasto. 
We thus consider planar homeomorphisms $f: \mathbb{R}^{2} \rightarrow \mathbb{R}^{2}$ so that $f \in$ $W_{\text {loc }}^{1,1}\left(\mathbb{R}^{2} ; \mathbb{R}^{2}\right)$ with $\|D f(x)\|^{2} \leq K(x) J_{f}(x)$ a.e. where $\exp (\lambda K)$ is locally integrable for some $\lambda>0$. For short, let us call such an $f$ a mapping of locally exponentially integrable distortion. Let us write $B$ for the unit disc and $S$ for the unit circle. Recall that a homeomorphism $f: B \rightarrow f(B) \subset \mathbb{R}^{2}$ is quasiconformal if $f \in W_{\text {loc }}^{1,1}\left(B ; \mathbb{R}^{2}\right)$ and the above distortion inequality holds for some $K$ with $1 \leq K(x) \leq K$, almost everywhere in the unit disc. Very little is known about $f(S)$ under a quasiconformal mapping with an extension to a mapping of locally exponentially integrable distortion. Necessarily $f(S)$ is a Jordan curve, and based on the work of David [1] the area of $f(S)$ is zero. In fact, $f(S)$ can have Hausdorff dimension two, but the generalized gauge dimension of $f(S)$ is bounded away from two [7], [14]. In this paper we consider the shape of $f(S)$. In the quasiconformal case, the three point condition rules out cusps from $f(S)$, but cusps can be formed in our more general setting [5]. By a cusp we mean a curve of the type $|y|=x^{1+s}$, $0<x<1 / 100$, where $s>0$. Fixing a model Jordan domain $\Omega_{s}$ whose boundary contains this cusp but is otherwise smooth (see Section 2), our main result reads as follows.

Theorem 1. Let $f: B \rightarrow \Omega_{s}$ be a quasiconformal mapping and assume that $\hat{f}: \mathbb{R}^{2} \rightarrow \mathbb{R}^{2}$ is a homeomorphic extension of $f$. If $\hat{f}$ is a mapping of finite distortion, then

$$
\int_{2 B} \exp (\lambda K(x)) d x=\infty \quad \text { for all } \lambda>1 / s .
$$

Conversely, there exists a quasiconformal mapping $f: B \rightarrow \Omega_{s}$, which extends to a homeomorphic mapping of finite distortion $\hat{f}: \mathbb{R}^{2} \rightarrow \mathbb{R}^{2}$ with

$$
\int_{2 B} \exp (\lambda K(x)) d x<\infty \quad \text { for all } \lambda<1 / s .
$$

Theorem 1 can be viewed as the first step towards understanding the extension problem in the category of mappings of locally exponentially integrable distortion. We would like to see general sufficient geometric conditions for the extendability.

In the course of the proof of Theorem 1, we establish an essentially sharp modulus of continuity for the mappings in question. This complements the earlier results for mappings of locally exponentially integrable distortion $[\mathbf{9}],[\mathbf{8}]$ and $[\mathbf{1 1}]$. 
Theorem 2. Let $f: \mathbb{R}^{2} \rightarrow \mathbb{R}^{2}$ be a homeomorphic mapping of finite distortion such that $\exp (\lambda K(x)) \in L^{1}(2 B)$ for some $\lambda>0$. If the restriction of $f$ to the open unit disc $B$ is quasiconformal, then for any $\varepsilon>0$ there exist positive constants $\hat{C}$ and $\tilde{C} \geq 2$ such that

$$
|f(x)-f(y)| \leq \frac{\hat{C}}{\log ^{\frac{\lambda}{1+\varepsilon}} \frac{\tilde{C}}{|x-y|}},
$$

whenever $x, y \in \bar{B}$.

If we drop the assumption that $f$ be quasiconformal in $B$, then the sharp exponent of the logarithm in (1) is $\lambda / 2$, see [11]. The mapping $f$ referred to in the second part of Theorem 1 does not satisfy (1) with any negative $\varepsilon$. Thus Theorem 2 is rather sharp. We would like to know if Theorem 2 holds with $\varepsilon=0$, and if one even could obtain a modulus of continuity of the type $o\left(\log ^{-\lambda} \frac{1}{|x-y|}\right)$. This would allow one to include the case $\lambda=1 / s$ in the first part of Theorem 1 .

Notice that, in Theorem 1, we consider mappings that are quasiconformal in the unit disc. The setting where $f$ is only assumed to be of locally exponentially integrable distortion is dealt with in a forthcoming paper [12].

\section{Definitions}

In this paper we consider the mappings of finite distortion on the plane. By plane we mean either $\mathbb{R}^{2}$ or the complex plane $\mathbb{C}$, depending on which is more convenient for the situation. In general, we will perform the computations in $\mathbb{R}^{2}$; the complex plane $\mathbb{C}$ and moreover the extended complex plane (i.e. the Riemann sphere) $\overline{\mathbb{C}}=\mathbb{C} \cup\{\infty\}$ are mainly used to simplify the definition of certain mappings.

Let $\Omega \subset \mathbb{R}^{2}$ be a domain, i.e. a connected and open subset of $\mathbb{R}^{2}$. We say that a homeomorphism $f: \Omega \rightarrow f(\Omega) \subset \mathbb{R}^{2}$ has finite distortion if the following conditions are satisfied:

1. $f \in W_{\mathrm{loc}}^{1,1}\left(\Omega ; \mathbb{R}^{2}\right)$

2. $\|D f(x)\|^{2} \leq K(x) J_{f}(x)$ a.e. $x \in \Omega$

for some measurable function $K(x) \geq 1$ which is finite almost everywhere. The function $K(x)$ is referred as a distortion (function) of $f$ and the phrase exponentially integrable distortion means that $\exp (\lambda K(x)) \in$ $L_{\text {loc }}^{1}(\Omega)$ for some $\lambda>0$.

Above $D f(x)$ denotes the differential matrix of $f$ at the point $x$ (which for $f \in W_{\text {loc }}^{1,1}$ exists a.e.) and $J_{f}(x):=\operatorname{det} D f(x)$ is the Jacobian. The 
norm of $D f(x)$ is defined as

$$
\|D f(x)\|:=\max \left\{|D f(x) e|: e \in \mathbb{R}^{2},|e|=1\right\} .
$$

If $E \subset \mathbb{R}^{2}$ is a measurable set of positive and finite area, $0<|E|<\infty$, we denote the mean integral of a measurable function $f: \mathbb{R}^{2} \rightarrow \mathbb{R}$ over $E$ by

$$
f_{E} f(x) d x:=\frac{1}{|E|} \int_{E} f(x) d x .
$$

We denote by $B(x, r)$ the open disc of radius $r>0$ centered at $x \in \mathbb{R}^{2}$ and write $B:=B(0,1)$. The boundary of a set $A$ is denoted by $\partial A$ and the closure by $\bar{A}$. Furthermore, we denote the image of a plane set $A$ in the standard polar transformation by $P_{A}$. For example, when $R>0$ and $0<\alpha_{2}-\alpha_{1} \leq 2 \pi$, we have

$$
P_{\left[0, R[\times] \alpha_{1}, \alpha_{2}[\right.}=\left\{(r \cos \theta, r \sin \theta) \in \mathbb{R}^{2}: 0 \leq r<R, \alpha_{1}<\theta<\alpha_{2}\right\}
$$

and

$$
P_{\{R\} \times] \alpha_{1}, \alpha_{2}[}=\left\{(R \cos \theta, R \sin \theta) \in \mathbb{R}^{2}: \alpha_{1}<\theta<\alpha_{2}\right\} .
$$

One of the main tools used in this paper is the following modulus of a path family $\Gamma$, see $[\mathbf{9}],[\mathbf{1 3}]$. Let $E$ and $F$ be subsets of $\Omega \subset \mathbb{R}^{2}$ and let $\Gamma$ consist of all locally rectifiable paths joining $E$ to $F$ in $\Omega$. We set

$$
\begin{array}{r}
\bmod (\Gamma, \Omega)=\inf \left\{\int_{\Omega} \rho^{2}(x) d x: \rho: \mathbb{R}^{2} \rightarrow[0, \infty[\text { is a Borel function }\right. \\
\text { s.t. } \left.\int_{\gamma} \rho d s \geq 1 \text { for every } \gamma \in \Gamma\right\},
\end{array}
$$

and by $\bmod _{K(x)}(\Gamma, \Omega)$ we mean the $K(x)$-weighted modulus, where instead of $\int \rho^{2}(x) d x$ we take the infimum over $\int \rho^{2}(x) K(x) d x$.

For a domain $\Omega \subset \mathbb{R}^{2}$ the quasihyperbolic distance between points $x_{1}, x_{2} \in \Omega$ is defined in the usual way by setting

$$
k_{\Omega}\left(x_{1}, x_{2}\right)=\inf _{\gamma} \int_{\gamma} \frac{d s}{\operatorname{dist}(x, \partial \Omega)},
$$

where the infimum is taken over all rectifiable arcs $\gamma$ joining $x_{1}$ to $x_{2}$ in $\Omega$. The minimizing arcs are called quasihyperbolic geodesics and the existence of such a minimizer is guaranteed by a result of Gehring and Osgood [4]. A rectifiable arc that has an endpoint at the boundary of $\Omega$ is called a quasihyperbolic geodesic if its every subarc in $\Omega$ is a quasihyperbolic qeodesic. 
Finally, we define the domains $\Omega_{s} \subset \mathbb{R}^{2}$ for $s>0$ by setting

$$
\Omega_{s}=\left\{\left(x_{1}, x_{2}\right) \in \mathbb{R}^{2}: 0<x_{1}<1,\left|x_{2}\right|<x_{1}^{1+s}\right\} \cup B\left(x_{s}, r_{s}\right),
$$

where $x_{s}=(s+2,0)$ and $r_{s}=\sqrt{(s+1)^{2}+1}$. Figure 1 shows the boundary of $\Omega_{2}$.

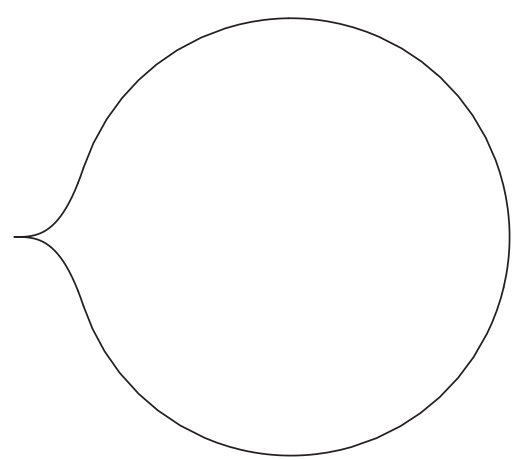

Figure 1 . The boundary of the domain $\Omega_{s}$ with $s=2$.

Then $\Omega_{s}$ is domain with a cusp, where the parameter $s$ determines the degree of the cusp. Notice that $\Omega_{s}$ is clearly not a quasidisc, because the three point condition fails (only) at the tip of the cusp. Thus if we have a conformal or quasiconformal mapping $f: B \rightarrow \Omega_{s}$, it does not extend to a quasiconformal mapping of the entire plane. On the other hand, if we allow the extension to be a homeomorphism of finite distortion, we have the result given by Theorem 1 .

\section{Proofs}

The first part of Theorem 1 follows from the following more general theorem that states a sufficient condition for the form of the cusp by the means of the quasihyperbolic distance $k_{f(B)}$ in the set $f(B)$.

Theorem 3. Let $f: \mathbb{R}^{2} \rightarrow \mathbb{R}^{2}$ be a homeomorphic mapping of finite distortion such that $\exp (\lambda K(x)) \in L^{1}(2 B)$ for some $\lambda>0$ and that the restriction of $f$ to $B$ is quasiconformal. If there exists a point $x_{0} \in \partial B$ such that for points $f(x)$ near $f\left(x_{0}\right) \in \partial f(B)$ that lie on the quasihyperbolic geodesic connecting the points $f(0)$ and $f\left(x_{0}\right)$ in $f(B)$

$$
k_{f(B)}(f(0), f(x)) \geq C\left|f(x)-f\left(x_{0}\right)\right|^{-s}
$$

for some $s>0$ and $C>0$, then $\lambda \leq 1 / s$. 
To prove Theorem 3 we need to prove Theorem 2 first. To this end, by combining some ideas from $[\mathbf{9}]$ and $[\mathbf{1 1}]$, we prove the following technical lemma.

Lemma 1. Let $i_{1}$ and $i_{2}$ be integers such that $i_{1}+1<i_{2}<0$ and

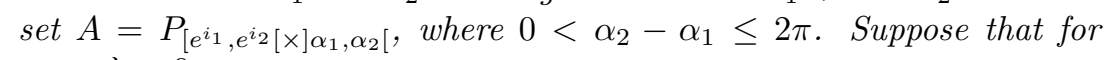
some $\lambda>0$

$$
I:=\int_{2 B} \exp (\lambda K(x)) d x<\infty
$$

where $K(x) \geq 1$ is measurable.

Set

$$
\varphi(s)=\frac{\lambda}{s \log \left(\frac{I e^{6}}{\left(\alpha_{2}-\alpha_{1}\right) s^{2}}\right)}
$$

for $s>0$, and define

$$
\rho(x)= \begin{cases}\varphi(|x|)\left(\int_{e^{i_{1}}}^{e^{i_{2}}} \varphi(s) d s\right)^{-1} & \text { for } e^{i_{1}}<|x|<e^{i_{2}} \\ 0 & \text { otherwise }\end{cases}
$$

Then

$$
\int_{A} \rho^{2}(x) K(x) d x \leq\left(\alpha_{2}-\alpha_{1}\right)\left(1+g\left(i_{2}\right)\right)\left(\int_{e^{i_{1}}}^{e^{i_{2}}} \varphi(s) d s\right)^{-1}
$$

where $g(i)=8 \log ^{-1}\left(\frac{I e^{6}}{\left(\alpha_{2}-\alpha_{1}\right) e^{2 i}}\right)$.

Proof: By using Fubini's theorem we see that

(4) $\int_{A} \rho^{2}(x) K(x) d x=\sum_{i=i_{1}}^{i_{2}-1} \int_{e^{i}}^{e^{i+1}} \int_{P_{\{t\} \times] \alpha_{1}, \alpha_{2}[}} \rho^{2}(x) K(x) d \sigma d t$

$$
=\frac{1}{\left(\int_{e^{i_{1}}}^{e^{i_{2}}} \varphi(s) d s\right)^{2}} \sum_{i=i_{1}}^{i_{2}-1} \int_{e^{i}}^{e^{i+1}} \int_{P_{\{t\} \times] \alpha_{1}, \alpha_{2}[}} \varphi^{2}(|x|) K(x) d \sigma d t
$$

where $d \sigma$ is the length element of the circle $\partial B_{t}$. 
Applying a change of variable and using the fact that $\varphi^{2}\left(e^{s}\right) e^{2 s}$ is increasing when $s<0$ we conclude that

$$
\begin{aligned}
\int_{e^{i}}^{e^{i+1}} & \int_{P_{\{t\} \times] \alpha_{1}, \alpha_{2}[}} \varphi^{2}(|x|) K(x) d \sigma d t \\
& =\left(\alpha_{2}-\alpha_{1}\right) \int_{e^{i}}^{e^{i+1}} \varphi^{2}(t) t \int_{P_{\{t\} \times] \alpha_{1}, \alpha_{2}[}} K(x) d \sigma d t \\
& =\left(\alpha_{2}-\alpha_{1}\right) \int_{i}^{i+1} \varphi^{2}\left(e^{s}\right) e^{2 s} \int_{P_{\left.\left\{e^{s}\right\} \times\right] \alpha_{1}, \alpha_{2}[}} K(x) d \sigma d s \\
& \leq\left(\alpha_{2}-\alpha_{1}\right) \varphi^{2}\left(e^{i+1}\right) e^{2(i+1)} \int_{i}^{i+1} f_{P_{\left.\left\{e^{s}\right\} \times\right] \alpha_{1}, \alpha_{2}[}} K(x) d \sigma d s
\end{aligned}
$$

Applying Jensen's inequality twice we obtain

$$
\begin{aligned}
\int_{i}^{i+1} & f_{P_{\left.\left\{e^{s}\right\} \times\right] \alpha_{1}, \alpha_{2}}[} K(x) d \sigma d s \\
& \leq \int_{i}^{i+1} \frac{1}{\lambda} \log \left(f_{P_{\left.\left\{e^{s}\right\} \times\right] \alpha_{1}, \alpha_{2}[}} \exp (\lambda K(x)) d \sigma\right) d s \\
& \leq \frac{1}{\lambda} \log \left(\int_{i}^{i+1} f_{P_{\left.\left\{e^{s}\right\} \times\right] \alpha_{1}, \alpha_{2}[}} \exp (\lambda K(x)) d \sigma d s\right)
\end{aligned}
$$

(6)

and by a straightforward calculation one sees that

$$
\int_{i}^{i+1} f_{P_{\left.\left\{e^{s}\right\} \times\right] \alpha_{1}, \alpha_{2}[}} \exp (\lambda K(x)) d \sigma d s \leq \frac{I e^{6}}{\left(\alpha_{2}-\alpha_{1}\right) e^{2 i}} .
$$

Combining (4), (5), (6) and (7) we arrive at

(8) $\int_{A} \rho^{2}(x) K(x) d x$

$$
\begin{aligned}
\leq \frac{\left(\alpha_{2}-\alpha_{1}\right)}{\left(\int_{e^{i_{1}}}^{e^{i_{2}}} \varphi(s) d s\right)^{2}} \sum_{i=i_{1}}^{i_{2}-1} \varphi^{2}\left(e^{i+1}\right) e^{2(i+1)} \frac{1}{\lambda} \log \left(\frac{I e^{6}}{\left(\alpha_{2}-\alpha_{1}\right) e^{2 i}}\right) \\
=\frac{\left(\alpha_{2}-\alpha_{1}\right)}{\left(\int_{e^{i_{1}}}^{e^{i_{2}}} \varphi(s) d s\right)^{2}} \sum_{i=i_{1}}^{i_{2}-1} \frac{\lambda \log \left(\frac{I e^{6}}{\left(\alpha_{2}-\alpha_{1}\right) e^{2 i}}\right)}{\log ^{2}\left(\frac{I e^{6}}{\left(\alpha_{2}-\alpha_{1}\right) e^{2(i+1)}}\right)} .
\end{aligned}
$$


As $i<0, \alpha_{2}-\alpha_{1} \leq 2 \pi$ and $I \geq 4 \pi$, we have that

$$
2 \log ^{-1}\left(\frac{I e^{6}}{\left(\alpha_{2}-\alpha_{1}\right) e^{2 i}}\right) \leq 2 \log ^{-1}\left(2 e^{6}\right)<\frac{1}{3} \text {. }
$$

Using the fact that $\left(\frac{1}{1-t}\right)^{2} \leq 1+4 t$ for $0<t<\frac{1}{3}$, we readily obtain the estimate

$$
\begin{aligned}
& \log \left(\frac{I e^{6}}{\left(\alpha_{2}-\alpha_{1}\right) e^{2 i}}\right) / \log ^{2}\left(\frac{I e^{6}}{\left(\alpha_{2}-\alpha_{1}\right) e^{2(i+1)}}\right) \\
&=\left(\frac{1}{1-2 \log ^{-1}\left(\frac{I e^{6}}{\left(\alpha_{2}-\alpha_{1}\right) e^{2 i}}\right)}\right)^{2} \frac{1}{\log \left(\frac{I e^{6}}{\left(\alpha_{2}-\alpha_{1}\right) e^{2 i}}\right)} \\
& \leq\left(1+8 \log ^{-1}\left(\frac{I e^{6}}{\left(\alpha_{2}-\alpha_{1}\right) e^{2 i_{2}}}\right)\right) \frac{1}{\log \left(\frac{I e^{6}}{\left(\alpha_{2}-\alpha_{1}\right) e^{2 i}}\right)} \\
&=:\left(1+g\left(i_{2}\right)\right) \frac{1}{\log \left(\frac{I e^{6}}{\left(\alpha_{2}-\alpha_{1}\right) e^{2 i}}\right)} .
\end{aligned}
$$

On the other hand,

$$
\text { (10) } \begin{aligned}
& \sum_{i=i_{1}}^{i_{2}-1} \frac{\lambda}{\log \left(\frac{I e^{6}}{\left(\alpha_{2}-\alpha_{1}\right) e^{2 i}}\right)} \leq \sum_{i=i_{1}}^{i_{2}-1} \int_{i}^{i+1} \frac{\lambda d s}{\log \left(\frac{I e^{6}}{\left(\alpha_{2}-\alpha_{1}\right) e^{2 s}}\right)} \\
= & \int_{i_{1}}^{i_{2}} \frac{\lambda d s}{\log \left(\frac{I e^{6}}{\left(\alpha_{2}-\alpha_{1}\right) e^{2 s}}\right)}=\int_{e^{i_{1}}}^{e^{i_{2}}} \frac{\lambda d t}{t \log \left(\frac{I e^{6}}{\left(\alpha_{2}-\alpha_{1}\right) t^{2}}\right)}=\int_{e^{i_{1}}}^{e^{i_{2}}} \varphi(s) d s .
\end{aligned}
$$

Finally, combining (8), (9) and (10) gives us the desired conclusion

$$
\int_{A} \rho^{2}(x) K(x) d x \leq\left(\alpha_{2}-\alpha_{1}\right)\left(1+g\left(i_{2}\right)\right)\left(\int_{e^{i_{1}}}^{e^{i_{2}}} \varphi(s) d s\right)^{-1} .
$$

Proof of Theorem 2: We will first prove the desired modulus of continuity on the boundary $\partial B$. To simplify the notation we consider the translated unit disc $\tilde{B}:=B((1,0), 1)$ instead of $B$.

Let $\varepsilon>0$ be given and assume that $0<R<1 / e^{3}$. Let $x, y \in \partial \tilde{B}$ with $y \in B\left(x, R / e^{3}\right)$. By choosing $E \subset \partial \tilde{B}$ to be the shortest arc joining $x$ to $y$, we obtain a continuum $E$ with $\operatorname{diam}(E)=|x-y|<R / e^{3}$. Furthermore, we may assume without loss of generality that $0 \in E$ and we set $r:=\operatorname{diam}(E)$. Next we fix an arc $F \subset \partial \tilde{B}$ by setting $F=$ 
$\partial \tilde{B} \cap B((2,0), 1 / 2)$. Because $f$ is a homeomorphism, the images $f(E):=$ $E^{\prime}$ and $f(F):=F^{\prime}$ of the clearly separate continua $E$ and $F$ are also separate continua.

We denote by $\Gamma$ and $\Gamma^{\prime}$ the path families connecting $E$ to $F$ and $E^{\prime}$ to $F^{\prime}$, respectively. First, using a modulus inequality from [9] and then a standard modulus estimate (cf. [3, pp. 183-184] and [10, Chapter II, $\S 2]$ ), we obtain the lower bound

$$
\bmod _{K(x)}\left(\Gamma, \mathbb{R}^{2}\right) \geq \bmod \left(\Gamma^{\prime}, \mathbb{R}^{2}\right) \geq \frac{2 \pi}{\log \left(\frac{C_{1}}{\operatorname{diam}(f(E))}\right)}
$$

where the constant $C_{1}$ depends on $\operatorname{dist}(f(E), f(F))$ which is bounded from below and from above as $f(F)$ and $f(\tilde{B})$ were fixed.

As now $0<r<R / e^{3}$, we may pick integers $i_{1}$ and $i_{2}$ such that

$$
i_{1}-1<\log r \leq i_{1}, \quad i_{2} \leq \log R<i_{2}+1 \quad \text { and } \quad i_{1}+1<i_{2}<0 .
$$

To get an upper bound, we observe that if $\rho$ is defined as in (3) we have

$$
\bmod _{K(x)}\left(\Gamma, \mathbb{R}^{2}\right) \leq \int_{B\left(0, e^{i_{2}}\right) \backslash B\left(0, e^{i_{1}}\right)} \rho^{2}(x) K(x) d x
$$

because $\int_{\gamma} \rho d s \geq 1$ for each $\gamma \in \Gamma$. Next we define the angles $\alpha_{1}$ and $\alpha_{2}$ by setting $\alpha_{1}=\pi / 2-e^{i_{2}}$ and $\alpha_{2}=2 \pi-\alpha_{1}$ and split $B\left(0, e^{i_{2}}\right) \backslash B\left(0, e^{i_{1}}\right)$ into $P_{\left[e^{i_{1}}, e^{i_{2}}\left[\times\left[\alpha_{1}, \alpha_{2}\right]\right.\right.}$ and $P_{] e^{i_{1}}, e^{i_{2}}[\times]-\alpha_{1}, \alpha_{1}[}$. The choice of $\alpha_{1}$ and $\alpha_{2}$ assures that $P_{]} e^{i_{1}, e^{i_{2}}[\times]-\alpha_{1}, \alpha_{1}[} \subset \tilde{B}$. Now, by Lemma 1 , we have that

$$
\int_{P_{\left[e^{i_{1}}, e^{i_{2}}[\times] \alpha_{1}, \alpha_{2}[\right.}} \rho^{2}(x) K(x) d x \leq\left(\pi+2 e^{i_{2}}\right)\left(1+g\left(i_{2}\right)\right) \frac{2}{\lambda} \Phi_{i_{2}}\left(i_{1}\right),
$$

where

$$
\Phi_{i_{2}}\left(i_{1}\right)=\log ^{-1}\left(\log \frac{I e^{6}}{\left(\pi+2 e^{i_{2}}\right) e^{2 i_{1}}} / \log \frac{I e^{6}}{\left(\pi+2 e^{i_{2}}\right) e^{2 i_{2}}}\right) .
$$

Next we estimate the integral of $\rho^{2}(x) K(x)$ over $P_{] e^{i_{1}}, e^{i_{2}}[\times]-\alpha_{1}, \alpha_{1}[}$. Notice that inside the $\operatorname{disk} \tilde{B}$ the distortion $K(x)$ is by assumption bounded by a constant $K$. By using the facts that $\varphi(s) s$ is increasing 
on $\left[e^{i_{1}}, e^{i_{2}}\right]$ and that $g\left(i_{2}\right)=\frac{8}{\lambda} \varphi\left(e^{i_{2}}\right) e^{i_{2}}$ we compute

$$
\begin{aligned}
& \int_{P_{] e^{i_{1}}, e^{i_{2}}[\times]-\alpha_{1}, \alpha_{1}[}} \rho^{2}(x) K(x) d x \\
& \leq \frac{K}{\left(\int_{e^{i_{1}}}^{e^{i_{2}}} \varphi(s) d s\right)^{2}} \int_{e^{i_{1}}}^{e^{i_{2}}} \int_{P_{\{t\} \times]-\alpha_{1}, \alpha_{1}[}} \varphi^{2}(t) d \sigma d t \\
& =\frac{2 \alpha_{1} K}{\left(\int_{e^{i_{1}}}^{e^{i_{2}}} \varphi(s) d s\right)^{2}} \int_{e^{i_{1}}}^{e^{i_{2}}} \varphi^{2}(t) t d t \\
& \leq 2 \alpha_{1} K \varphi\left(e^{i_{2}}\right) e^{i_{2}}\left(\int_{e^{i_{1}}}^{e^{i_{2}}} \varphi(s) d s\right)^{-1} \\
& \leq \frac{K}{4}\left(\pi-2 e^{i_{2}}\right) g\left(i_{2}\right) \Phi_{i_{2}}\left(i_{1}\right)
\end{aligned}
$$

where $\Phi_{i_{2}}\left(i_{1}\right)$ is defined as in (14). Combining (12), (13) and (15) we finally arrive at

(16) $\bmod _{K(x)}\left(\Gamma, \mathbb{R}^{2}\right) \leq\left(\left(\pi+2 e^{i_{2}}\right)\left(1+g\left(i_{2}\right)\right) \frac{2}{\lambda}+\frac{K}{4}\left(\pi-2 e^{i_{2}}\right) g\left(i_{2}\right)\right) \Phi_{i_{2}}\left(i_{1}\right)$.

Now, for any $\varepsilon>0$, by taking $R \geq e^{i_{2}}$ small enough at the beginning of the proof, we deduce from (16) that

$$
\bmod _{K(x)}\left(\Gamma, \mathbb{R}^{2}\right) \leq \frac{2 \pi(1+\varepsilon)}{\lambda} \log ^{-1}\left(\frac{1}{C_{2}} \log \frac{C_{3}}{(\operatorname{diam}(E))^{2}}\right)
$$

where $C_{3} \geq 2 e^{4}$ and $C_{2}$ depends only on $R$. Moreover, by combining (17) with (11), we obtain

$$
\log \left(\frac{C_{1}}{\operatorname{diam}(f(E))}\right) \geq \frac{\lambda}{1+\varepsilon} \log \left(\frac{1}{C_{2}} \log \frac{C_{3}}{(\operatorname{diam}(E))^{2}}\right) .
$$

Observing that $|f(x)-f(y)| \leq \operatorname{diam}(f(E))$ and $|x-y|=\operatorname{diam}(E)$, we conclude that whenever $|x-y| \leq R / e^{3}$, the desired estimate holds. Because $f(\partial \tilde{B})$ is bounded, this estimate follows for all $x, y \in \partial \tilde{B}$.

Next we show that this kind of an estimate is true also on $B$. Given $x \in B$ set $B_{1}=B\left(x, \frac{1-|x|}{2}\right)$ and fix a segment $I \subset \partial B$ such that 
$\operatorname{diam} I=\operatorname{diam} B_{1}$ and $\operatorname{dist}\left(B_{1}, \partial B\right)=\operatorname{dist}\left(B_{1}, I\right)$. From the basic properties of quasiconformal mappings it follows (see [4]) that for some constant $C>0$

$$
\frac{|f(x)-f(y)|}{\operatorname{diam} f\left(B_{1}\right)} \leq C\left(\frac{|x-y|}{\operatorname{diam} B_{1}}\right)^{1 / K},
$$

whenever $y \in B_{1}$ and

$$
\operatorname{diam} f\left(B_{1}\right) \asymp \operatorname{dist}\left(f\left(B_{1}\right), \partial \Omega\right),
$$

where $\Omega=f(B)$. If $\Gamma$ denotes the path family connecting $B_{1}$ to $I$ in $B$, then because $\operatorname{diam} B_{1}=\operatorname{diam} I$ and $2 \operatorname{dist}\left(B_{1}, I\right)=1-|x|=\operatorname{diam} B_{1}$, the modulus $\bmod (\Gamma, B)$ has a positive lower bound. Thus if $\Gamma^{\prime}$ is the path family connecting $f\left(B_{1}\right)$ to $f(I)$ in $\Omega$ then $\bmod \left(\Gamma^{\prime}, \Omega\right)$ has also a positive lower bound. This and (20) imply that

$$
\operatorname{dist}\left(f\left(B_{1}\right), f(I)\right) \leq C \operatorname{diam} f\left(B_{1}\right)
$$

and

$$
\operatorname{diam} f\left(B_{1}\right) \leq C \operatorname{diam} f(I),
$$

for some constant $C>0$ as otherwise $\bmod \left(\Gamma^{\prime}, \Omega\right)$ would be arbitrarily small.

We first consider points $x, y \in B$ that are relatively close to each other, i.e.

$$
y \in B\left(x, \frac{1-|x|}{2}\right) \quad \text { or } \quad x \in B\left(y, \frac{1-|y|}{2}\right) .
$$

Because of the symmetry, we may assume that $y \in B_{1}$. Combining (19) and (22) and using the previous result that an estimate of the type (1) holds on the boundary, we obtain

$$
\begin{aligned}
|f(x)-f(y)| & \leq \hat{C}_{1}\left(\frac{|x-y|}{\operatorname{diam} B_{1}}\right)^{1 / K} \operatorname{diam} f(I) \\
& \leq \hat{C}_{2}\left(\frac{|x-y|}{1-|x|}\right)^{1 / K} \log ^{-\frac{\lambda}{1+\varepsilon}} \frac{C_{3}}{1-|x|} \leq \hat{C}_{2} \log ^{-\frac{\lambda}{1+\varepsilon}} \frac{C_{3}}{|x-y|} .
\end{aligned}
$$

The last inequality follows from the facts that $0<|x-y| \leq 1-|x|<1$ and that the function $t \log ^{\frac{\lambda K}{1+\varepsilon}} \frac{C_{3}}{t}$ is increasing when $0<t<1$ as now $C_{3} \geq 2 e^{4}>1$.

Next we will consider points $x, y \in B$ that are relatively far from each other, i.e. neither of (23) holds. If this is the case, then we have that

$$
|x-y| \geq \max \left\{\frac{1-|x|}{2}, \frac{1-|y|}{2}\right\} \text {. }
$$


Let $x^{\prime}$ and $y^{\prime}$ denote the projections of $x$ and $y$ on the boundary $\partial B$. In the case $x=0$ choose $x^{\prime}=(1,0)$ and likewise with $y$. By applying the triangle inequality and $(24)$ we notice that

$$
\left|x^{\prime}-y^{\prime}\right| \leq\left|x-x^{\prime}\right|+|x-y|+\left|y-y^{\prime}\right| \leq 5|x-y| .
$$

As $x^{\prime}, y^{\prime} \in \partial B$ we may again use the estimate on the boundary to obtain

$$
\left|f\left(x^{\prime}\right)-f\left(y^{\prime}\right)\right| \leq \hat{C}_{1} \log ^{-\frac{\lambda}{1+\varepsilon}} \frac{C_{3}}{\left|x^{\prime}-y^{\prime}\right|} \leq \hat{C}_{1} \log ^{-\frac{\lambda}{1+\varepsilon}} \frac{\tilde{C}_{3}}{|x-y|},
$$

where $\tilde{C}_{3} \geq e^{3}$. Next we show that

$$
\left|f(x)-f\left(x^{\prime}\right)\right| \leq \hat{C}_{2} \log ^{-\frac{\lambda}{1+\varepsilon}} \frac{\tilde{C}_{3}}{|x-y|}
$$

If $B_{1}=B\left(x, \frac{1-|x|}{2}\right)$ and $I \subset \partial B$ are chosen as before, we may in addition assume that $x^{\prime} \in I$. Using (21) and (22) we see that

$$
\begin{aligned}
\left|f(x)-f\left(x^{\prime}\right)\right| & \leq \operatorname{dist}(f(x), f(I))+\operatorname{diam} f(I) \\
& \leq \operatorname{dist}\left(f\left(B_{1}\right), f(I)\right)+\operatorname{diam} f\left(B_{1}\right)+\operatorname{diam} f(I) \\
& \leq C \operatorname{diam} f(I),
\end{aligned}
$$

for some constant $C>0$. Again by estimating on the boundary and using the fact that $\operatorname{diam} I=1-|x|=\left|x-x^{\prime}\right| \leq 2|x-y|$, the inequality (26) readily follows. Exchanging the pair $x, x^{\prime}$ with $y, y^{\prime}$ gives the same estimate for $\left|f(y)-f\left(y^{\prime}\right)\right|$ and thus finally with (25) we have that

$$
\begin{aligned}
|f(x)-f(y)| & \leq\left|f(x)-f\left(x^{\prime}\right)\right|+\left|f\left(x^{\prime}\right)-f\left(y^{\prime}\right)\right|+\left|f(y)-f\left(y^{\prime}\right)\right| \\
& \leq \hat{C} \log ^{-\frac{\lambda}{1+\varepsilon}} \frac{\tilde{C}_{3}}{|x-y|} .
\end{aligned}
$$

From these two cases $(x, y \in \partial B$ or $x, y \in B)$ one easily obtains the remaining case when for example $x \in B$ and $y \in \partial B$ by using the triangle inequality.

Proof of Theorem 3: Let $\varepsilon>0$. Because $f$ is quasiconformal in $B$, there exists a constant $C_{0}>1$ such that

$$
k_{f(B)}(f(0), f(x)) \leq C_{0} k_{B}(0, x) .
$$

Let $\gamma_{0}$ denote the quasihyperbolic geodesic connecting the points $f(0)$ and $f\left(x_{0}\right)$. From Theorem 6.1. in [6] it follows that for all $x \in f^{-1}\left(\gamma_{0}\right)$ 
we have $\left|x_{0}-x\right| \asymp 1-|x|$. Thus with Theorem 2 we obtain for all $x \in$ $f^{-1}\left(\gamma_{0}\right)$ sufficiently close to $x_{0}$ that

$$
\left|f(x)-f\left(x_{0}\right)\right| \leq \frac{C}{\log ^{\frac{\lambda}{1+\varepsilon}}\left(1 /\left|x-x_{0}\right|\right)} \leq \frac{\hat{C}}{\log \frac{\lambda}{1+\varepsilon}(1 /(1-|x|))} .
$$

Combining (2), (27), (28) and the fact that the quasihyperbolic distance in $B$ satisfies

we conclude that

$$
k_{B}(0, x)=\log \frac{1}{1-|x|},
$$

$$
\log \frac{1}{1-|x|} \geq \tilde{C}\left(\log \frac{1}{1-|x|}\right)^{\frac{\lambda}{1+\varepsilon} s}
$$

for those $x \in f^{-1}\left(\gamma_{0}\right)$ for which $f(x)$ is near $f\left(x_{0}\right)$. Because we may take $x$ arbitrarily close to $x_{0}$ along $f^{-1}\left(\gamma_{0}\right)$ (making $|x| \rightarrow 1$ ) it must be that

$$
\frac{\lambda}{1+\varepsilon} s \leq 1,
$$

from which the claim follows by letting $\varepsilon \rightarrow 0$.

Proof of Theorem 1: One quite easily observes in the following way that $\Omega_{s}$ satisfies the condition (2) of Theorem 3. Let $\gamma_{0}$ be a quasihyperbolic qeodesic in $\Omega_{s}$ joining the origin (the tip of the cusp) to a point $\tilde{x} \in \Omega_{s}$. Notice that by the symmetric form of the boundary, the qeodesic $\gamma_{0}$ must travel very close to the $x_{1}$-axis (in comparison to the boundary) as it approaches origin. So for points $x=\left(x_{1}, x_{2}\right)$ near the origin on the geodesic $\gamma_{0}$ we have that

$$
\begin{aligned}
k_{\Omega_{s}}(\tilde{x}, x) \asymp k_{\Omega_{s}}\left((1,0),\left(x_{1}, 0\right)\right) & =\int_{x_{1}}^{1} \frac{d t}{\operatorname{dist}\left((t, 0), \partial \Omega_{s}\right)} \\
& \asymp \int_{x_{1}}^{1} \frac{d t}{t^{1+s}} \asymp|x|^{-s},
\end{aligned}
$$

and the first claim of Theorem 1 follows.

For the second claim, we will construct for a given $s>0$ a homeomorphism $f$ of finite distortion that satisfies the conditions given in Theorem 1 . This construction was initially motivated by the conformal mapping $f_{s}: B \rightarrow \mathbb{C}$

$$
f_{s}(z)=\left(1-\log \left(\frac{z+1}{2}\right)\right)^{-\frac{1}{s}}
$$


and its behaviour near the point -1 , where the boundary of $f_{s}(B)$ actually resembles the boundary of $\Omega_{s}$.

We begin by mapping the unit $\operatorname{disc} B$ conformally onto the open right half plane $H_{R}:=\left\{\left(x_{1}, x_{2}\right) \in \mathbb{R}^{2}: x_{1}>0\right\}$ so that the point $(-1,0) \in \partial B$ maps to the origin. Using the complex notation we define this mapping $f_{1}: \overline{\mathbb{C}} \rightarrow \overline{\mathbb{C}}$ as $f_{1}(z)=(z+1) /(1-z)$.

Next, we set

$$
g(r)=\log ^{-1}(2 / r)
$$

and define linear functions $\left.L_{r}^{i}:\right]-\frac{\pi}{2}, \frac{\pi}{2}\left[\rightarrow \mathbb{R}\right.$ and $L_{r}^{o}:\left[\frac{\pi}{2}, \frac{3 \pi}{2}\right] \rightarrow \mathbb{R}$ by setting

$$
L_{r}^{i}(\theta)=\frac{2 \arctan g(r)}{\pi}(\theta+\pi / 2)-\arctan g(r)
$$

and

$$
L_{r}^{o}(\theta)=\frac{2 \pi-2 \arctan g(r)}{\pi}(\theta-\pi / 2)+\arctan g(r) .
$$

Let $x \in \mathbb{R}^{2}$ satisfy $|x| \leq 1$. We may represent $x$ in the polar coordinates, $x=(r \cos \theta, r \sin \theta)$, so that $0 \leq r \leq 1$ and either $\theta \in]-\frac{\pi}{2}, \frac{\pi}{2}[$ or $\theta \in\left[\frac{\pi}{2}, \frac{3 \pi}{2}\right]$. Using this notation we define the mapping $f_{2}: \mathbb{R}^{2} \rightarrow \mathbb{R}^{2}$ in polar coordinates on $\bar{B}$ by setting

$$
f_{2}(r, \theta)= \begin{cases}\left(G_{s}(r), L_{r}^{i}(\theta)\right) & \text { if } 0<r \leq 1 \text { and } \theta \in]-\frac{\pi}{2}, \frac{\pi}{2}[ \\ \left(G_{s}(r), L_{r}^{o}(\theta)\right) & \text { if } 0<r \leq 1 \text { and } \theta \in\left[\frac{\pi}{2}, \frac{3 \pi}{2}\right] \\ 0 & \text { if } r=0\end{cases}
$$

where $G_{s}(r)=g^{1 / s}(r) \sqrt{1+g^{2}(r)}$. Outside the closed unit disc $\bar{B}$, the mapping $f_{2}$ will be defined in a bilipschitz manner. First we define $h: S(0,1) \rightarrow S\left(0, G_{s}(1)\right)$ by setting $h(x)=f_{2}(x)$ on the unit circle $S:=S(0,1)=\partial \bar{B}(0,1)$. The mapping $h$ is clearly a bilipschitz mapping on $S$. Next we set

$$
f_{2}(x)=|x| h\left(\frac{x}{|x|}\right) \quad \text { if } x \in \mathbb{R}^{2} \backslash \bar{B} .
$$

A simple calculation shows that if $h$ is an $L$-bilipschitz mapping, then $f_{2}$ will also be an $L$-bilipschitz mapping on $S(0, R)$ for all $R \geq 1$. This and the fact that $\left|f_{2}(x)\right|=|x| G_{s}(1)$ for all $|x| \geq 1$ assures that $f_{2}$ will be a bilipschitz mapping on $\mathbb{R}^{2} \backslash \bar{B}$ and the bilipschitz constant of $f_{2}$ depends only on $L$ and $G_{s}(1)$.

The definition in (29) gives a mapping that maps the line segment $\left\{\left(x_{1}, x_{2}\right) \in \mathbb{R}^{2}: x_{1}=0,-1 \leq x_{2} \leq 1\right\}$ to the set $\left\{\left(x_{1}, x_{2}\right) \in \mathbb{R}^{2}\right.$ : 
$\left.\left|x_{2}\right|=x_{1}^{1+s}, 0 \leq x_{1} \leq G_{s}(1)\right\}$, thus forming a cusp of the correct degree. Also, $\left\{\left(x_{1}, x_{2}\right) \in \mathbb{R}^{2}: x_{1}=0,\left|x_{2}\right|>1\right\}$ gets mapped to the set $\left\{\left(x_{1}, x_{2}\right) \in \mathbb{R}^{2}: x_{1}>G_{s}(1),\left|x_{2}\right|=x_{1}\right\}$. What we have now is an unbounded domain with a cusp at the origin and whose boundary is a piecewise smooth curve. There are only three points where the smoothnes fails, the origin (zero angle) and the points $\left(G_{s}(1), G_{s}^{1+s}(1)\right)$ and $\left(G_{s}(1),-G_{s}^{1+s}(1)\right)$ (non-zero angles). These two non-zero angles have the size $3 \pi / 4-\arctan \left((1+s) G_{s}^{s}(1)\right)$.

Next we make this cusp domain bounded by mapping the right half plane onto the disc $B((1 / 2,0), 1 / 2)$ with the mapping $f_{3}: \overline{\mathbb{C}} \rightarrow \overline{\mathbb{C}}, f_{3}(z)=$ $z /(z+1)$ and denote $\tilde{\Omega}_{s}:=f_{3}\left(f_{2}\left(f_{1}(B)\right)\right)$. The mapping $f_{3}$ will somewhat alter the shape of the cusp at the origin, but not essentially as it will be seen. On the other hand, being conformal, it will preserve the size of the two aforementioned non-zero angles and will create one more non-zero angle at the point $(1,0)$, of the size $\pi / 2$. Otherwise the boundary curve will still be smooth. Because of these facts, there exists a sense preserving bilipschitz mapping $f_{4}: \mathbb{R}^{2} \rightarrow \mathbb{R}^{2}$ for which $f_{4}\left(\tilde{\Omega}_{s}\right)=\Omega_{s}$ and moreover: this mapping $f_{4}$ can be chosen so that for some bounded function $L(x, y): \mathbb{R}^{2} \rightarrow[1, L], 1<L<\infty$, we have that

$$
\frac{1}{L(x, y)}|x-y| \leq\left|f_{4}(x)-f_{4}(y)\right| \leq L(x, y)|x-y|
$$

and $L(x, y) \rightarrow 1$ when $|x|+|y| \rightarrow 0$. To justify this last claim we notice that for $0<r_{0}<G_{s}(1)$ we have by a straightforward calculation that

$$
\begin{aligned}
\partial \tilde{\Omega}_{s} \cap B\left(0, r_{0}\right)= & \left\{p^{-1}(t)\left(t^{2+2 s}+t^{2}+t, t^{1+s}\right) \in \mathbb{R}^{2}: 0 \leq t<r_{0}\right\} \\
& \cup\left\{p^{-1}(t)\left(t^{2+2 s}+t^{2}+t,-t^{1+s}\right) \in \mathbb{R}^{2}: 0 \leq t<r_{0}\right\},
\end{aligned}
$$

where $p(t):=t^{2+2 s}+t^{2}+2 t+1$. If we consider the upper half plane we see from (32) that near the origin the boundary points of $\tilde{\Omega}_{s}$ are of the form $\left(t+o\left(t^{2}\right), t^{1+s}+o\left(t^{2+s}\right)\right)$ and likewise on the lower half plane. Thus the bilipschitz correction of $\tilde{\Omega}_{s}$ to $\Omega_{s}$ can be done in the manner explained before.

Finally we will set $f: \mathbb{R}^{2} \rightarrow \mathbb{R}^{2}, f(x)=f_{4} \circ f_{3} \circ \tilde{f}_{2} \circ f_{1}(x)$. Here $\tilde{f}_{2}$ is the homeomorphic extension of $f_{2}$ to $\overline{\mathbb{C}}$ obtained by setting $\tilde{f}_{2}(x)=f_{2}(x)$ when $x$ is finite and $\tilde{f}_{2}(\infty)=\infty$. This definition clearly gives us a sense preserving homeomorphism for which $f(B)=\Omega_{s}$.

Next we show that the distortion function of $f$ satisfies the required conditions. In fact, it will be enough to compute the distortion of $f_{2}$, because the conformal mappings $f_{1}$ and $f_{3}$ do not give any contribution to it and the contribution of the bilipschitz mapping $f_{4}$ will be in fact a 
bounded multiplier that goes to one when we approach the origin. The latter claim follows from the facts that the distortion of a $L$-bilipschitz mapping is $L^{2}$, and $f_{4}$ satisfies the inequality (31).

Outside the unit disc $B$ the mapping $f_{2}$ is quasiconformal, as it is seen directly from the definition to be sense preserving and bilipschitz there. Hence we need to show that $f_{2}$ is quasiconformal in $B \cap H_{R}$ and has an exponentially integrable distortion in $B \backslash H_{R}$. To show this, we consider a point $x \in B$, which we again represent in the form $x=(r \cos \theta, r \sin \theta)$, such that $0 \leq r<1$ and either $\theta \in]-\frac{\pi}{2}, \frac{\pi}{2}\left[\right.$ or $\theta \in\left[\frac{\pi}{2}, \frac{3 \pi}{2}\right]$. We first consider the case $\theta \in]-\frac{\pi}{2}, \frac{\pi}{2}\left[\right.$, i.e. the set $B \cap H_{R}$.

To compute $K_{f_{2}}(x)$, we choose a local coordinate system at every point $x \in B$ by setting $e_{1}^{x}=(\cos \theta, \sin \theta)$ and $e_{2}^{x}=(-\sin \theta, \cos \theta)$. Notice that the vector $e_{1}^{x}$ points to the radial direction and $e_{2}^{x}$ is perpendicular to it, so it points to the angular direction. To this basis on the preimage side we associate a similar basis on the image side, denoting it by $\left(e_{1}^{f_{2}(x)}, e_{2}^{f_{2}(x)}\right)$. Figure 2 gives an example of the situation.
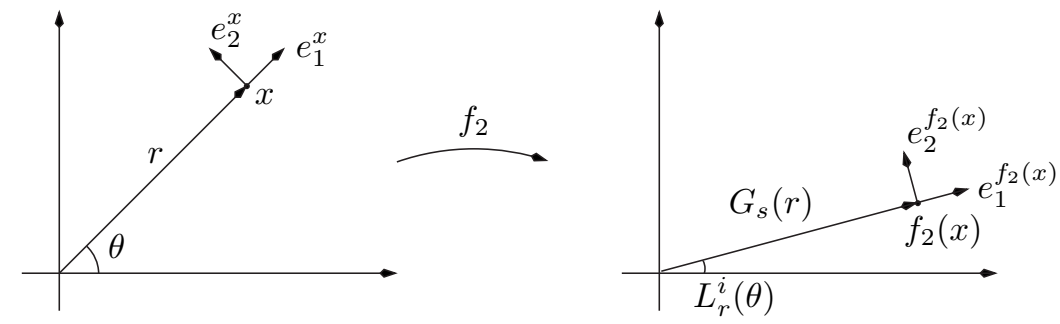

Figure 2. The choice of the local coordinates used to compute $D f_{2}(x)$

We now represent the differential matrix of $f_{2}$ at the point $x$ by using the bases $\left(e_{1}^{x}, e_{2}^{x}\right)$ and $\left(e_{1}^{f_{2}(x)}, e_{2}^{f_{2}(x)}\right)$ which we will refer from now on as $E_{x}$ and $E_{f_{2}(x)}$ respectively. The resulting differential matrix is

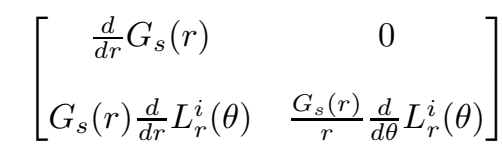

$=\left[\begin{array}{cc}\frac{1}{s r} g^{1 / s+1}(r) \sqrt{1+g^{2}(r)}+\frac{1}{r} \frac{g^{1 / s+3}(r)}{\sqrt{1+g^{2}(r)}} & 0 \\ \frac{2 \theta}{\pi r} \frac{g^{1 / s+2}(r)}{\sqrt{1+g^{2}(r)}} & \frac{2}{\pi r} g^{1 / s}(r) \sqrt{1+g^{2}(r)} \arctan g(r)\end{array}\right]$

and is computed as follows. 
As the radial part of $f_{2}$ depends only on $r$, the partial derivative of the first component of $f_{2}$ in the basis $E_{f_{2}(x)}$ in the $e_{1}^{x}$-direction is $\frac{d}{d r} G_{s}(r)$. The radial part does not depend on the angle, and so the partial derivative of the first component of $f_{2}$ in the basis $E_{f_{2}(x)}$ in the $e_{2}^{x}$-direction is zero.

Next we observe that for $\varepsilon>0$ the change in the angular part, and thus in the $e_{2}^{f_{2}(x)}$-direction, is $G_{s}(r)\left(L_{r+\varepsilon}^{i}(\theta)-L_{r}^{i}(\theta)\right)$ when the change to the $e_{1}^{x}$ direction is $\varepsilon$. Thus the partial derivative of the second component of $f_{2}$ in the basis $E_{f_{2}(x)}$ in the $e_{1}^{x}$-direction is

$$
\lim _{\varepsilon \rightarrow 0} \frac{G_{s}(r)\left(L_{r+\varepsilon}^{i}(\theta)-L_{r}^{i}(\theta)\right)}{\varepsilon}=G_{s}(r) \frac{d}{d r} L_{r}^{i}(\theta) .
$$

Similarly, the partial derivative of the second component of $f_{2}$ in the basis $E_{f_{2}(x)}$ in the $e_{2}^{x}$-direction is

$$
\lim _{\varepsilon \rightarrow 0} \frac{G_{s}(r)\left(L_{r}^{i}(\theta+\varepsilon)-L_{r}^{i}(\theta)\right)}{r \varepsilon}=\frac{G_{s}(r)}{r} \frac{d}{d \theta} L_{r}^{i}(\theta) .
$$

To estimate $K_{f_{2}}(x)$ from (33) we use the following known result (see [13]) which states that for a linear bijection $A: \mathbb{R}^{2} \rightarrow \mathbb{R}^{2}$ the distortion $K$ of $A=\left[\begin{array}{ll}a & b \\ c & d\end{array}\right]$ satisfies

$$
K+\frac{1}{K}=\frac{a^{2}+b^{2}+c^{2}+d^{2}}{|a d-b c|} .
$$

As $K \geq 1$ we get a simple upper estimate from (36) for $K$ :

$$
K \leq \frac{a^{2}+b^{2}+c^{2}+d^{2}}{|a d-b c|} .
$$

Thus by a straightforward calculation we obtain the estimate

$$
\begin{aligned}
K_{f_{2}}(x) \leq \frac{\pi}{2 s} \frac{g(|x|)}{\arctan g(|x|)} & +\frac{2 s}{\pi}\left(\frac{g(|x|)}{\arctan g(|x|)}\right)^{-1} \\
+ & \left(\frac{s \pi}{2} g^{2}(|x|)+\pi+\frac{2 s \theta^{2}}{\pi}\right) \frac{g^{3}(|x|)}{\arctan g(|x|)} .
\end{aligned}
$$

Because $g(|x|) /(\arctan g(|x|)) \rightarrow 1, g^{3}(|x|) /(\arctan g(|x|)) \rightarrow 0$ and $g^{2}(|x|) \rightarrow 0$ as $|x| \rightarrow 0$, and all terms are bounded from above when $|x|$ is bounded, we see that $f_{2}$ is quasiconformal also on the set $B \cap H_{R}$. 
Next we consider the case $\theta \in\left[\frac{\pi}{2}, \frac{3 \pi}{2}\right]$, i.e. $x \in B \backslash H_{R}$. Similar reasoning as in the first case gives the differential matrix

$$
\begin{aligned}
& (39)\left[\begin{array}{cc}
\frac{d}{d r} G_{s}(r) & 0 \\
G_{s}(r) \frac{d}{d r} L_{r}^{o}(\theta) & \frac{G_{s}(r)}{r} \frac{d}{d \theta} L_{r}^{o}(\theta)
\end{array}\right] \\
& =\left[\begin{array}{cc}
\frac{1}{s r} g^{1 / s+1}(r) \sqrt{1+g^{2}(r)}+\frac{1}{r} \frac{g^{1 / s+3}(r)}{\sqrt{1+g^{2}(r)}} & 0 \\
\left(2-\frac{2 \theta}{\pi}\right) \frac{1}{r} \frac{g^{1 / s+2}(r)}{\sqrt{1+g^{2}(r)}} & \left(2-\frac{2}{\pi} \arctan g(r)\right) \frac{1}{r} g^{1 / s}(r) \sqrt{1+g^{2}(r)}
\end{array}\right] .
\end{aligned}
$$

The estimate for $K_{f_{2}}(x)$ is

(40) $\quad K_{f_{2}}(x) \leq 2 s g^{-1}(|x|)-\frac{2 s}{\pi} g^{-1}(|x|) \arctan g(|x|)$

$$
+\left(\frac{1}{s}+s g^{4}(|x|)+2 g^{2}(|x|)+s\left(2-\frac{2 \theta}{\pi}\right)^{2} g^{2}(|x|)\right) \frac{g(|x|)}{2-\frac{2}{\pi} \arctan g(|x|)} .
$$

Now, as $g(|x|) \rightarrow 0$ when $|x| \rightarrow 0$ and thus also $\arctan g(|x|) \rightarrow 0$, the last term in (40) goes to zero. Moreover, as $g^{-1}(|x|) \arctan g(|x|) \rightarrow 1$ when $|x| \rightarrow 0$, the second term goes to $-2 s / \pi$. Thus it follows from (40) that there exists $0<r_{0}<1$ such that

$$
K_{f_{2}}(x) \leq 2 s \log (2 /|x|),
$$

for all $x \in B\left(0, r_{0}\right) \backslash H_{R}$. The fact that the distortion $K_{f_{2}}(x)$ is bounded for $r_{0} \leq|x| \leq 1$ and the estimate (41) show that $\exp \left(\lambda K_{f_{2}}(x)\right) \in L^{1}(B \backslash$ $H_{R}$ ) for all $\lambda<1 / s$. Thus we conclude that the distortion of $f_{2}$ satisfies the conditions given in Theorem 1.

As mentioned before, $f_{1}$ and $f_{3}$ did not give any contribution to the distortion of $f$ and the contribution of $f_{4}$ is a bounded multiplier that goes to one when one approaches the origin. Moreover, $f_{1}$ is bilipschitz in $f^{-1}\left(B\left(0, r_{0}\right)\right)$. Thus the distortion $K_{f}(x)$ of $f$ satisfies $\exp \left(\lambda K_{f}(x)\right) \in$ $L^{1}(2 B)$ for all $\lambda<1 / s$ and $K_{f}(x)$ is bounded on $B$.

Acknowledgements. Juhani Takkinen would like to thank Mika Leikas for several intriguing discussions and comments that helped to shape this article to its current form.

\section{References}

[1] G. David, Solutions de l'équation de Beltrami avec $\|\mu\|_{\infty}=1$, Ann. Acad. Sci. Fenn. Ser. A I Math. 13(1) (1988), 25-70. 
[2] F. W. Gehring, "Characteristic properties of quasidisks", Séminaire de Mathématiques Supérieures 84, Presses de l'Université de Montréal, Montreal, Que., 1982.

[3] F. W. Gehring and O. Martio, Quasiextremal distance domains and extension of quasiconformal mappings, J. Analyse Math. 45 (1985), 181-206.

[4] F. W. Gehring and B. G. Osgood, Uniform domains and the quasihyperbolic metric, J. Analyse Math. 36 (1979), 50-74 (1980).

[5] P. Haïssinsky, Chirurgie parabolique, C. R. Acad. Sci. Paris Sér. I Math. 327(2) (1998), 195-198.

[6] J. Heinonen And R. NÄKKI, Quasiconformal distortion on arcs, J. Anal. Math. 63 (1994), 19-53.

[7] D. Herron and P. Koskela, Mappings of finite distortion: gauge dimension of generalized quasicircles, Illinois J. Math. 47(4) (2003), $1243-1259$.

[8] P. Koskela and J. Onninen, Mappings of finite distortion: the sharp modulus of continuity, Trans. Amer. Math. Soc. 355(5) (2003), 1905-1920.

[9] P. Koskela and J. Onninen, Mappings of finite distortion: Capacity and modulus inequalities, J. reine angew. Math. 599 (2006), $1-26$.

[10] O. Lehto and K. I. ViRTAnen, "Quasiconformal mappings in the plane", Second edition, Translated from the German by K. W. Lucas, Die Grundlehren der mathematischen Wissenschaften 126, Springer-Verlag, New York-Heidelberg, 1973.

[11] J. Onninen And X. Zhong, A note on mappings of finite distortion: the sharp modulus of continuity, Michigan Math. J. 53(2) (2005), 329-335.

[12] J. TAKkinen, Mappings of finite distortion: formation of cusps II, (to appear).

[13] J. VÄıs̈̈LÄ, "Lectures on n-dimensional quasiconformal mappings", Lecture Notes in Mathematics 229, Springer-Verlag, BerlinNew York, 1971.

[14] S. ZAKeRI, David maps and Hausdorff dimension, Ann. Acad. Sci. Fenn. Math. 29(1) (2004), 121-138. 
Department of Mathematics and Statistics

University of Jyväskylä

P. O. Box 35

FI-40014 University of Jyväskylä

Finland

E-mail address: pkoskela@maths.jyu.fi

E-mail address: juhani@maths.jyu.fi

Primera versió rebuda el 28 de setembre de 2006, darrera versió rebuda el 27 de novembre de 2006. 